Open Access Full Text Article

\title{
Arsenic sulfide induces miR-4665-3p to inhibit gastric cancer cell invasion and migration
}

This article was published in the following Dove Press journal:

Drug Design, Development and Therapy

\author{
Xiuli Zhang* \\ Zhen Tan* \\ Ting Kang \\ Chuanying Zhu \\ Siyu Chen
}

Department of Oncology, Xin Hua Hospital, School of Medicine, Shanghai Jiao Tong University, Shanghai, People's Republic of China

*These authors contributed equally to this work
Correspondence: Siyu Chen

Department of Oncology, Xin Hua Hospital, School of Medicine, Shanghai

Jiao Tong University, 1665 Kongjiang

Road, Shanghai 200092, People's Republic of China

Tel +862I 25077642

Email siyu.chen@shsmu.edu.cn
Purpose: Gastric carcinogenesis is a multistep process and is the second-highest cause of cancer death worldwide with a high incidence of invasion and metastasis. MicroRNAs (miRNAs) engage in complex interactions with the machinery that controls the transcriptome and concurrently target multiple mRNAs. Recent evidence has shown that miRNAs are involved in the cancer progression, including promoting cell-cycle, conferring resistance to apoptosis, and enhancing invasiveness and metastasis. Here, we aim to elucidate the roles of miRNAs, especially microRNA-4665-3p (miR-4665-3p), in the inhibitory effect of arsenic sulfide in gastric cancer (GC).

Methods: The arsenic sulfide-induced miRNA expression alterations in AGS cells was determined by miRNA microarray. RT-PCR was used to further verify the arsenic sulfideregulated miRNAs in GC tissues. The inhibition of miR-4665-3p on the migration and invasion of GC cells were determined by wound healing assay and transwell assay. Western blot analysis was used to detect the expression of EMT related proteins and the putative target of miR-4665-3p.

Results: The miR-4665-3p was up-regulated by arsenic sulfide and showed inhibition upon the migration and invasion of GC cells. MiRBase and Western blotting indicated that miR4665-3p directly down-regulated the oncoprotein GSE1. Morphological observation also indicated that the up-regulation of miR-4665-3p inhibits the EMT in GC cells.

Conclusion: Our data demonstrates that the increased expression of miR-4665-3p induced by arsenic sulfide suppresses the cell invasion, metastasis and EMT of GC cells, and has the potential to be a novel therapeutic target in GC.

Keywords: arsenic sulfide, miR-4665-3p, gastric cancer, invasion, migration

\section{Introduction}

Gastric cancer (GC) is the second leading cause of cancer death and the fourth most common malignancy all over the world. ${ }^{1}$ Approximately $50 \%$ of all gastric cancers occurring in Asia, especially in China, Japan and Korea. ${ }^{2}$ Given that lacking of early diagnosed methods, many patients are unfortunately found at a late stage with extensive invasion and metastasis. And even with comprehensive systematic therapy, the patients with advanced-stage GC are still strongly related to poor prognosis, with the 5-year overall survival rate less than $20 \%{ }^{3}$ Although we have already known that Helicobacter pylori infection, as well as many genetic and environmental factors are all crucial to GC carcinogenesis and development. ${ }^{4-6}$ Considering the complex process of GC and the above unpleasant statistics, the molecular mechanisms of GC are of great importance and should to be further elucidated. 
Extensive research in recent years has indicated that miRNAs play an important role in the pathogenesis of GC. ${ }^{7,8}$ MiRNAs are endogenous non-coding RNAs including 22-25 nucleotides which function at the post-transcriptional level as negative regulators of gene expression. ${ }^{9-11}$ By binding to the 3'-untranslated regions (3'-UTRs) of target mRNAs, miRNAs cause the mRNA degradation or block the mRNA translation. ${ }^{10}$ As one of the key regulators of gene expression, miRNAs can modulate almost one-third of human genes ${ }^{12,13}$ and engage in a wide range of cellular biological functions, including proliferation, differentiation, apoptosis and tumorigenesis. $^{14,15}$ The cell migration and invasion, which can be regulated by the miRNAs, are significant to the progression of GC. Increased researches are now focused on the inhibitory effect of miRNAs upon the cell migration and invasion of GC cells. MiR-125a restrains cell migration and invasion by targeting STAT3 in GC cells. ${ }^{16}$ MiR-618 suppresses metastasis by downregulating the expression of TGF- $\beta 2$ in GC cells. ${ }^{17}$ Furthermore, miR-1254 inhibits cell migration and invasion by down-regulating Smurfl in GC cells. ${ }^{18}$ These findings have led to the recognition of the important role of miRNAs in inhibiting cell migration and invasion in GC and prompts to discover novel biomarkers in the diagnosis of GC. However, the biological functions and molecular mechanisms of miR-4665-3p in GC have not been reported. In this study, we found that miR-4665-3p can be up-regulated by arsenic sulfide and showed anti-tumor effect.

Previously, we reported that traditional Chinese medicine arsenic sulfide $\left(\mathrm{As}_{4} \mathrm{~S}_{4}\right)$ inhibits the cell invasion and migration in GC through impairing the ability of basement membrane destroying and meanwhile increasing the cell adhesion ability of GC cells. ${ }^{19}$ In this study, we aimed to investigate whether miRNAs are involved in the arsenicinduced cytotoxicity in GC. Our miRNA microarray analysis indicated that the miR-4665-3p increased significantly after $\mathrm{As}_{4} \mathrm{~S}_{4}$ treatment. Furthermore, in vitro experiments showed that the miR-4665-3p suppressed the GC cells invasion and migration through modulating GSE1. In addition, that expression of miR-4665-3p was lower in GC tissues compared with the normal adjacent gastric tissue.

These results offer us a deep understanding of the underlying molecular mechanism of arsenic sulfide against GC and provide a basis for miR-4665-3p as a potential therapeutic target for GC.

\section{Materials and methods \\ Cell culture and reagents}

The human gastric cancer cell lines AGS, MGC803 were obtained from the Cell Bank, Chinese Academy of Sciences (Shanghai, People's Republic of China). No ethics statement was required from the institutional review board for the use of these cell lines. AGS cells were cultured in DMEM/F12 1:1 medium (Hyclone, Logan, UT, USA) and MGC803cells were cultured in RPMI 1640 medium (Thermo Fisher Scientific, Waltham, MA, USA), both cells were incubated at $37^{\circ} \mathrm{C}$ in a humidified atmosphere of $95 \%$ air and $5 \% \mathrm{CO}_{2}$. All medium was supplemented with $10 \%$ fetal bovine serum (Thermo Fisher Scientific). Highly purified arsenic sulfide $\left(\mathrm{As}_{4} \mathrm{~S}_{4}\right)$ was prepared from mined natural realgar and the preparation of $\mathrm{As}_{4} \mathrm{~S}_{4}$ solution was performed as previously described. ${ }^{20}$ The stock solution of $\mathrm{As}_{4} \mathrm{~S}_{4}$ was $133.36 \mu \mathrm{M}$ and then was diluted to work solution with complete culture medium. MiRNA mimics were purchased from GenePharma (Shanghai, China). Antibodies for E-cadherin, Vimentin, SP1 and VEGF were purchased from Cell Signaling Technology (Beverly, MA). Antibodies for GSE1 was purchased from Abcam (Cambridge, MA) while antibodies for $\beta$ actin was obtained from Proteintech Group (Wuhan, China).

\section{RNA isolation}

For miRNA microarray or real-time polymerase chain reaction (PCR) analysis, total RNA was isolated using Trizol reagent (Invitrogen, Carlsbad, CA, USA) according to the manufacturer's instructions. Then Total RNA was quantified by the NanoDrop ND-2000 (Thermo Fisher Scientific) and the RNA integrity for miRNA microarray was assessed using Agilent Bioanalyzer 2100 (Agilent Technologies) by the OEbiotech company (Shanghai, China).

\section{miRNA microarray}

RNA extracted from AGS cells incubated with $1 \mu \mathrm{M} \mathrm{As}{ }_{4} \mathrm{~S}_{4}$ for $12 \mathrm{~h}$ was labeled as the tested group and RNA from untreated AGS cells served as control. Then the sample labeling, microarray hybridization and washing were performed based on the manufacturer's standard protocols by the Shanghai OEbiotech company. Briefly, total RNA was dephosphorylated, denaturated and then labeled with Cyanine-3-CTP. After purification, the labeled RNAs were hybridized onto the microarray. After washing, the arrays were scanned with the Agilent Scanner G2505C (Agilent Technologies). Feature Extraction software (version10.7.1.1, Agilent Technologies) was used to analyze 
array images to get raw data. Next, Genespring software (version 13.1, Agilent Technologies) was employed to finish the basic analysis with the raw data. To begin with, the raw data was normalized with the quantile algorithm. Differentially expressed miRNAs were then identified through fold change. The threshold set for up- and down-regulated genes was a fold change no less than 1.8 folds. Target genes of differentially expressed miRNAs were the intersection predicted with 3 databases (Targetscan, microRNAorg, PITA). GO analysis and KEGG analysis were applied to determine the roles of these target genes.

\section{Quantitative reverse transcription-PCR for miRNA analysis}

RNAs were isolated from cancer cells by Trizol reagent (Invitrogen). For analysis of miR-135b-5p, miR-4665-3p, miR-4739 and miR-6124 expression, Taqman MicroRNA Reverse Transcription Kit (Applied Biosystems, Foster City, CA, USA) was used for reverse transcription. U6 small nuclear RNA was used as internal control for normalization of miRNA expression. Amplification and analysis were performed on the Applied Biosystems 7500 sequence detection system (Applied Biosystems, FosterCity, CA, USA). Each trial was done in triplicate three times. Foldchange of miRNA in the samples was determined using the comparative threshold cycle $(\mathrm{Ct})$ method. And the primer sequences of targeted genes used in the polymerase chain reaction are listed in Table 2.

\section{miRNA transfection}

Transfection of miRNA mimics was performed using Lipofectamine 2000 (Invitrogen, Carlsbad, CA, USA) according to the manufacturer's instructions. Briefly, cells were seeded at a concentration of $5.0 \times 10^{4}$ cells per well for $24 \mathrm{~h}$ before transfection. Then, the oligomer-Lipofectamine 2000 complexes were diluted with the culture medium to the appropriate concentration. The culture medium was changed after $4-6 \mathrm{~h}$ and the cells were incubated at $37^{\circ} \mathrm{C}$ for $48 \mathrm{~h}$.

\section{Wound-healing assay}

Transfected cells (AGS and MGC803) were seeded in sixwell plates at a density of $5 \times 10^{5}$ cells per well. After transfection of $24 \mathrm{~h}$, the confluent monolayer in each well was scratched using $100 \mu \mathrm{l}$ pipette tips and washed with phosphate-buffered saline in order to remove the suspended cells. Then the medium containing $1 \%$ serum was used for cell culture. The assay was performed for 24 or $48 \mathrm{~h}$ at $37{ }^{\circ} \mathrm{C}$ and $5 \% \mathrm{CO}_{2}$. Wounds were photographed with a DMI3000 B inverted microscope (Leica Microsystems, Wetzlar, Germany) at $0 \mathrm{~h}, 24 \mathrm{~h}$, and $48 \mathrm{~h}$, and the migration rates of each group were calculated as previously described. ${ }^{19}$

\section{Transwell invasion assay}

Cell invasion assays were performed using 24-well transwell chambers (Corning Incorporated, Corning, NY, USA). Briefly, the transfected cells (AGS and MGC803) were cultured in the upper part of each chamber with $200 \mu \mathrm{L}$ DMEM/F12 1:1 and RPMI 1640 medium which contained 1\% serum respectively, and in the bottom of the Transwell chamber, $500 \mu \mathrm{L}$ of DMEM/F12 1:1 and RPMI 1640 medium with 10\% fetal bovine serum were added respectively. After cultivation at $37{ }^{\circ} \mathrm{C}$ and $5 \% \mathrm{CO}_{2}$ for $24 \mathrm{~h}$, non-vading cells was gently wiped with a cotton swab, and migrated cells were fixed with $4 \%$ paraformaldehyde for 20 mins at room temperature and then stained with crystal violet solution, followed by observation under a DMI3000 B inverted microscope. The invasion rates of each group were calculated according to previously described, invasion rate $(\%)=$ the number of the migrated cells in transfected group/the number of the migrated cells in control group $\times 100 \%{ }^{19}$

\section{Western blotting}

Total protein from the AGS and MGC803 cells was collected with enhanced RIPA lysis buffer (Beyotime, Shanghai, China) containing $1 \%$ dilution of the phenylmethanesulfonyl fluoride (PMSF) (Beyotime) on ice. Protein concentrations were determined using a microplate reader (Bio-TEK, USA) with a bicinchoninic acid protein assay kit (Beyotime). According to the molecular weight of the proteins, different concentrations of SDS polyacrylamide gels (Beyotime) were subjected to electrophoresis, and transferred to polyvinylidene fluoride membrane (Millipore, Billerica, MA, USA). After blocking nonspecific binding sites with $5 \%$ nonfat milk, the membrane was then incubated with diluted specific primary antibody at $4{ }^{\circ} \mathrm{C}$ overnight. Then after washed thrice in Tris-buffered saline and Tween 20 for 10 mins each time, the membrane was incubated with labeled secondary antibody at room temperature for $1 \mathrm{~h}$. The membrane was washed thrice again to remove the excessive secondary antibody. Finally, proteins were detected with ECL Plus kit (Beyotime) and were semiquantified using Image Lab software. 


\section{Patients and tissue samples}

The GC samples and their corresponding adjacent normal tissues were obtained from 30 patients diagnosed with GC and undergone surgery at the Xinhua Hospital Affiliated to Shanghai Jiao Tong University, School of Medicine (Shanghai, China). Tissues were immediately snap-frozen in liquid nitrogen after surgery and stored at $-80{ }^{\circ} \mathrm{C}$ until RNA extraction. All the patients enrolled provided written informed consent in accordance with the Declaration of Helsinki. This study was approved by the ethics committee of Xinhua Hospital Affiliated to Shanghai Jiao Tong University (XHEC-C-2017-262).

\section{Statistical analysis}

Experiments were repeated independently three times and all data was presented as the mean \pm standard deviation. Data were analyzed using one-way ANOVA followed by the least significant difference test (if the variance was equal) or the Games-Howell procedure (if the variance was unequal). $P<0.05$ was considered statistical significant. Statistical analysis was performed with SPSS software version 20.0 (IBM Corporation, Armonk, NY, USA) to evaluate the differences between groups.

\section{Results}

\section{The miRNA expression profile altered after $\mathrm{As}_{4} \mathrm{~S}_{4}$ treatment in AGS cells}

Table 1 presents the effect of $\mathrm{As}_{4} \mathrm{~S}_{4}$ on the miRNA expression profile in AGS cells. Among the altered miRNAs whose changes were above two folds compared to the controls, there are five miRNAs up-regulated and four were down-regulated in cells with $\mathrm{As}_{4} \mathrm{~S}_{4}$ treatment. The five up-

Table I Effects of $\mathrm{As}_{4} \mathrm{~S}_{4}$ on microRNA expression in AGS cells

\begin{tabular}{|l|l|}
\hline miRNA name & Fold change \\
\hline hsa-miR-6I24 & -12.4682 \\
hsa-miR-4788 & -9.56264 I \\
hsa-miR-940 & 2.7362304 \\
hsa-miR-4665-3p & 2.6765854 \\
hsa-miR-10a-5p & -2.30178 \\
hsa-miR-4430 & -2.28863 \\
hsa-miR-4739 & 2.2726707 \\
hsa-miR-65I5-3p & 2.0959923 \\
hsa-miR-135b-5p & 2.022177 \\
hsa-miR-130a-3p & 1.981393 \\
hsa-miR-107 & -1.8486315 \\
\hline
\end{tabular}

Notes: AGS cells were subjected to mRNA microarray after incubation with I $\mu \mathrm{M}$ $\mathrm{As}_{4} \mathrm{~S}_{4}$ for $12 \mathrm{~h}$. Cells untreated were used as control. The miRNA with the fold change exceeds 1.8 were listed. regulated miRNAs were hsa-miR-940, hsa-miR-4665-3p, hsa-miR-4739, hsa-miR-6515-3p, hsa-miR-135b-5p, and the four down-regulated miRNAs were hsa-miR-6124, hsa-miR-4788, hsa-miR-10a-5p, hsa-miR-4430. The miRNA expression difference induced by $\mathrm{As}_{4} \mathrm{~S}_{4}$ has also been changed into heat map (See Figure 1).

\section{Quantitation of miRNA expression by quantitative reverse transcription-PCR}

To further verify the results obtained from the microarray analysis, quantitative reverse transcription-PCR was performed. The above-mentioned miRNAs of AGS cells treated with $\mathrm{As}_{4} \mathrm{~S}_{4}$ for 2, 6, 12 and $24 \mathrm{~h}$ were compared with the untreated cells. The relative expression alteration of four of them in the $\mathrm{As}_{4} \mathrm{~S}_{4}$-treated cells compared with untreated cells was found to be consistent with the microarray analysis, namely hsa-miR-135b-5p, hsa-miR-4665-3p, hsa-miR4739, hsa-miR-6124 (Figure 2). The primers used were shown in Table 2. Furthermore, the results in gastric cancer cells MGC803 and colon cancer cells HCT116 were consistent with the results obtained in AGS cells (Figure S1).

\section{miR-4665-3p was down-regulated in GC tissues}

To fully investigate the role of these miRNAs in the progression of GC, we next collected 30 pairs of GC samples and the corresponding adjacent normal tissues to detect their expression levels between these two groups. The expression level of miR-4665-3p was significantly lower in GC samples than that in corresponding adjacent normal tissues (Figure 3A, $P<0.05$ ). Interestingly, when $20 \mathrm{GC}$ samples were stratified on the basis of the stage of gastric cancer, we observed that miR-4665-3p expression was further significantly down-regulated in latestage GC samples compared with those early-stage ones (Figure 3B, $P<0.05)$. Late-stage cancer cells get easier access to invade and migrate. However, no statistically significance between GC tissues and adjacent normal tissues were observed among the other three miRNAs. In general, these findings indicate that miR-4665-3p serve as a tumor suppressor and plays a pivotal role in the metastasis and progression of GC.

\section{miR-4665-3p suppressed the migration and invasion of GC cells}

To further investigate the function of miR-4665-3p in the metastasis of GC, the effect of miR-4665-3p on cell migration and invasion were detected by wound healing assay and transwell invasion assay, respectively. The studies of 


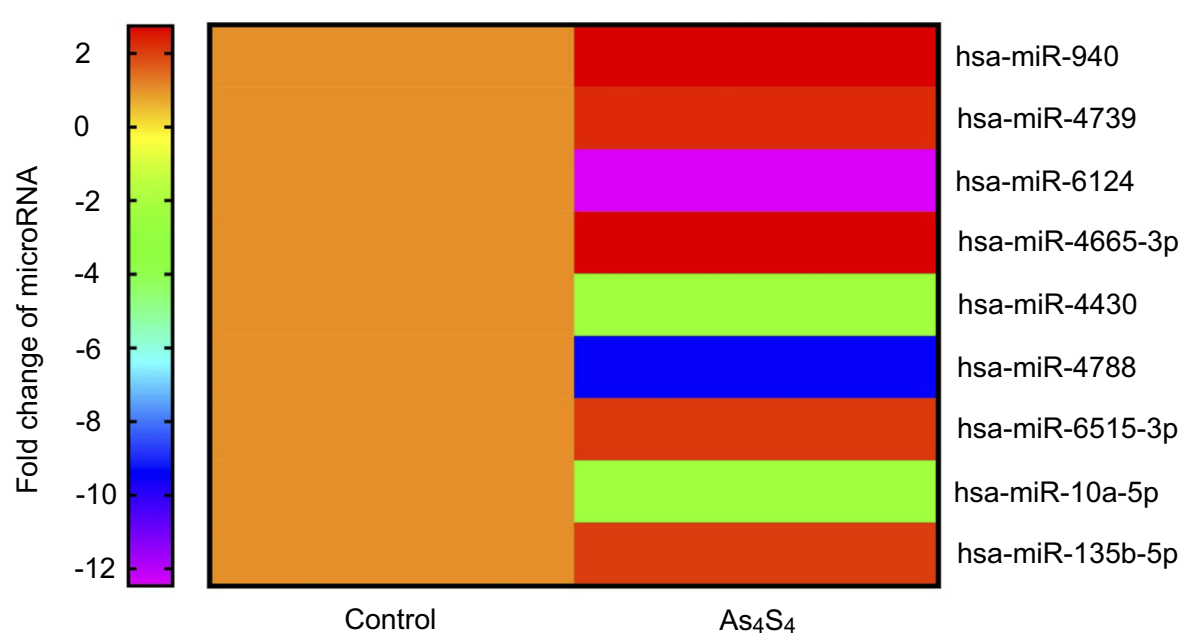

Figure I The heat map of miRNA expression difference induced by $\mathrm{As}_{4} \mathrm{~S}_{4}$ in AGS cells.
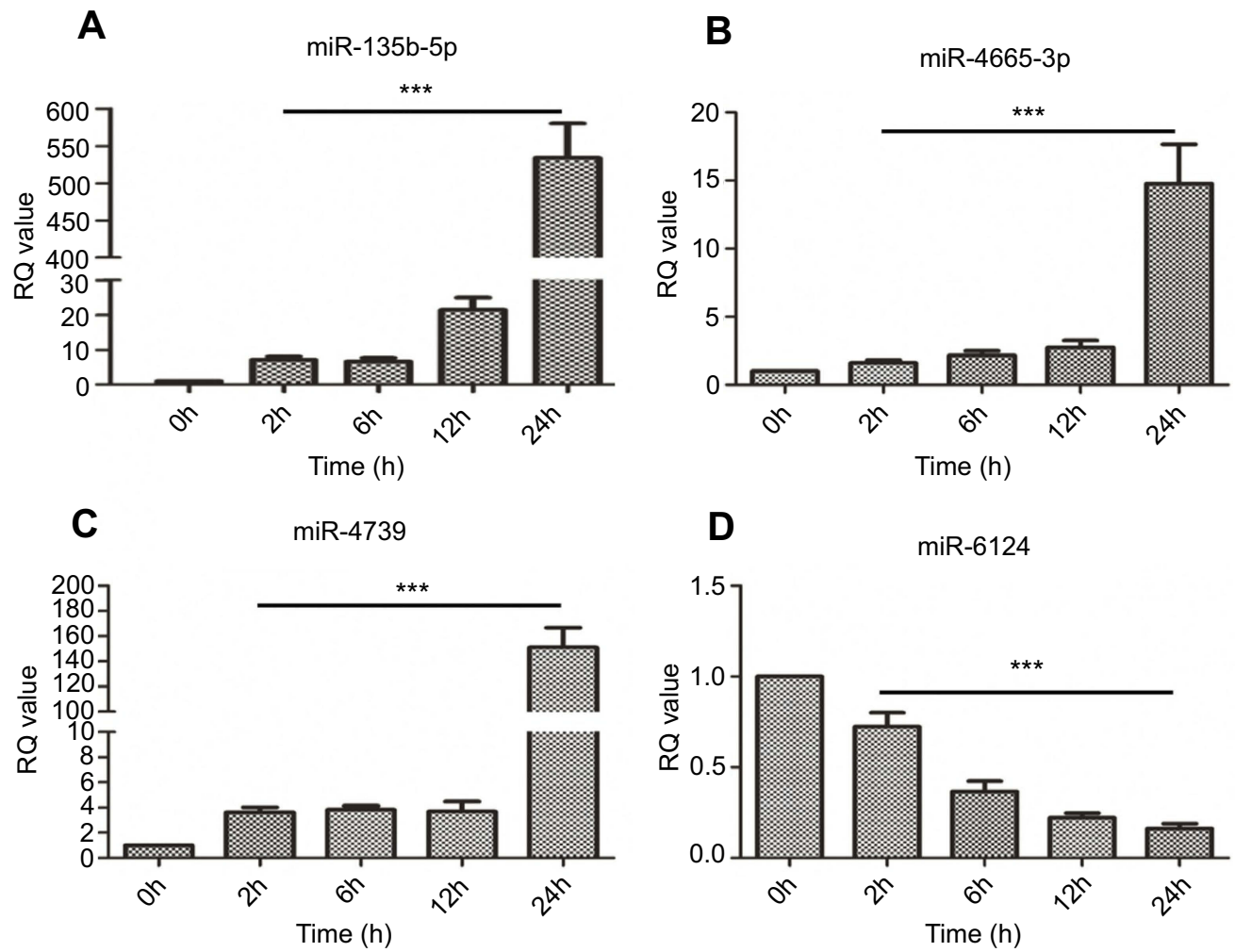

Figure 2 The alteration of miRNAs after treatment with $\mathrm{As}_{4} \mathrm{~S}_{4}$ in AGS cells.

Notes: (A) miR-135b-5p, (B) miR-4665-3p and (C) miR-4739 are up-regulated while (D) miR-6I 24 is down-regulated after treatment with As $S_{4}$ in $A G S$ cells. $* * * P<0.00 I$.

miR-4665-3p were conducted using a transient transfection strategy with miRNA mimics. It was found that the migration (Figure 4A and B) and invasion (Figure 4C and D) of AGS and MGC803 cells, which were infected with miR-4665-3p mimics, were significantly inhibited compared with the miRNA negative control. Collectively, these results indicated that miR-4665-3p suppressed the invasion and migration of GC cells.

\section{miR-4665-3p expression reverses the epithelial-mesenchymal transition of GC cells}

When AGS and MGC803 cells were transfected with miR4665-3p mimics, a typical morphological change was observed in the cells. The shape of cells transfected with miR-4665-3p mimics changed from the spindle-shaped, 
Table 2 Primer sequences for real-time polymerase chain reaction

\begin{tabular}{|l|l|}
\hline miRNA name & Primer sequence (5'-3') \\
\hline U6 & F: CTCGCTTCGGCAGCACA \\
& R: AACGCTTCACGAATTTGCGT \\
has-miR-135b-5p & GTA TGG CTT TTC ATT CCT ATG TG \\
has-miR-4665-3p & AAC TCG GCC GCG GCG C \\
has-miR-4739 & AAG GGA GGA GGA GCG GAG \\
has-miR-6I24 & TGG GAA AAG GAA GGG GGA G \\
\hline
\end{tabular}

Abbreviations: F, forward; R, reverse.

mesenchymal-like morphology of typical tumor cells to the round or cobblestone-like morphology of epithelial cells. Many of them accumulate together in groups, thereby enhancing cell-to-basal adhesion and reducing their ability to invade and migrate (Figure 5A and B). These results indicate that miR-4665-3p is involved in the epithelial-mesenchymal transition (EMT) of GC cells and it has been well established that EMT is essential for the tumor cells to enhance the capability of migration and invasion. $^{21}$ Therefore, we detected the expression of EMTrelated proteins including E-cadherin, Vimentin, SP1 and VEGF with Western blot analysis. The expression level of E-cadherin was significantly increased while Vimentin, SP1 and VEGF were decreased in miR-4665-3p-mimictreated GC cells (Figure 5C and D). These observations indicate that miR-4665-3p inhibits the EMT of GC cells.

\section{GSEI is a critical mediator of miR-4665- $3 p$ effects}

To further investigate the underlying molecular mechanism of miR-4665-3p in inhibiting the migration of GC cells, we

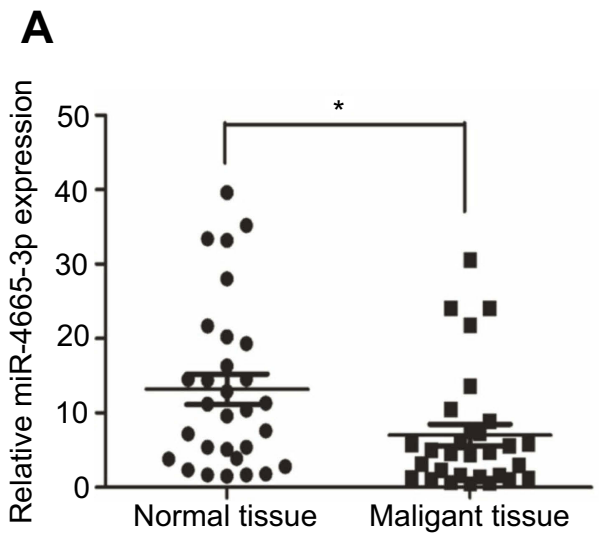

used TargetScan, miRBase to anticipate putative proteincoding gene targets of miR-4665-3p. Basing on these analyses, four candidate genes, namely GSE1, MKRN2, GATA2 and RCAN3, were selected for further investigation. We performed Western blot to verify whether miR-4665-3p can directly regulate the expression of these putative genes. The results showed that miR-4665-3p decreased the expression of GSE1 in AGS cells (Figure 6A and B), indicating that GSE1 might be a potential target gene of miR-4665-3p. The analysis diagram of the sequence complementary relationship between miR-4665-3p and GSE-1 was shown in Table 3. Furthermore, recent studies prove that GSE-1 plays a key role in promoting the migration and invasion of tumor cells in $\mathrm{GC},{ }^{22}$ which are in consistency with our current results.

\section{Discussion}

Metastasis, one of the most pivotal hallmarks of cancer, is the leading cause of cancer-related deaths worldwide. Thus, it's urgent to identify the metastatic factors and reveal the molecular mechanisms underlying cancer progression. Previously, in vitro and in vivo experiments have shown that $\mathrm{As}_{4} \mathrm{~S}_{4}$ can restrain the migration and invasion of GC cells. ${ }^{19}$ miRNAs play an important role in tumor initiation and progression. Recent evidence has also indicated that miRNAs engage in carcinogenesis and metastasis. ${ }^{23}$ By targeting STAT3, miR-125a restrains the cell migration and invasion in GC. ${ }^{16}$ MiR-618 was reported to suppress the metastasis of GC by downregulating the expression of TGF- $\beta 2 .^{17}$ In addition, miR-1254 showed inhibitory effects upon the cell migration, and invasion of GC cells by down-regulating Smurf1. ${ }^{18}$ Hence, conforming tumor-related miRNAs and their direct

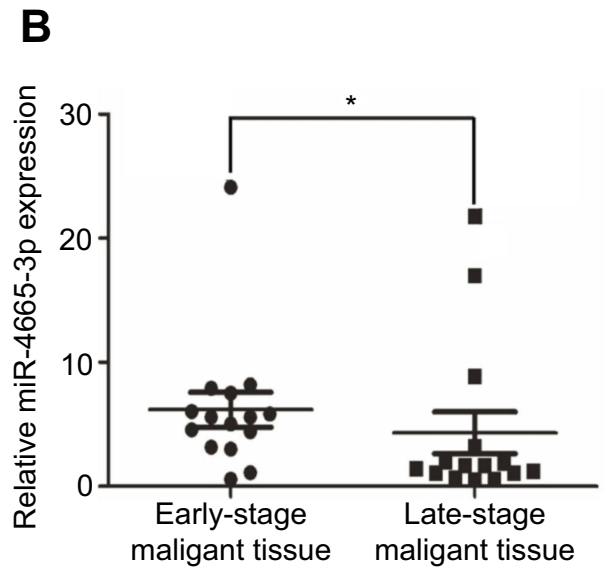

Figure 3 The expression of miR-4665-3p in GC tissues.

Notes: (A) The expression of miR-4665-3p was relatively lower in malignant tissues compared to normal tissues in GC. (B) There is less expression level of miR-4665-3p in late-stage maligant tissues relatively to early-stage maligant tissues in GC. $* P<0.05$. 
A

AGS

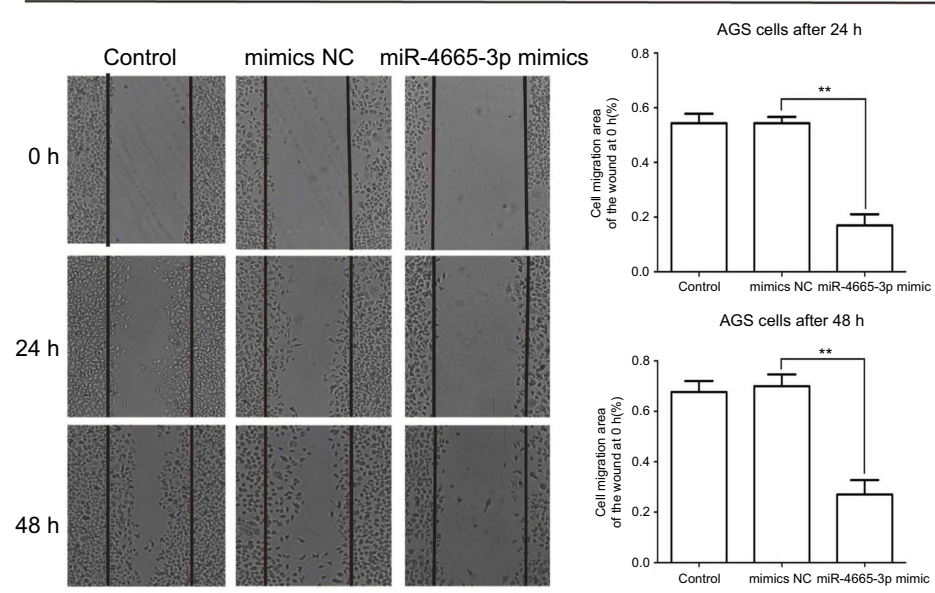

B

MGC803
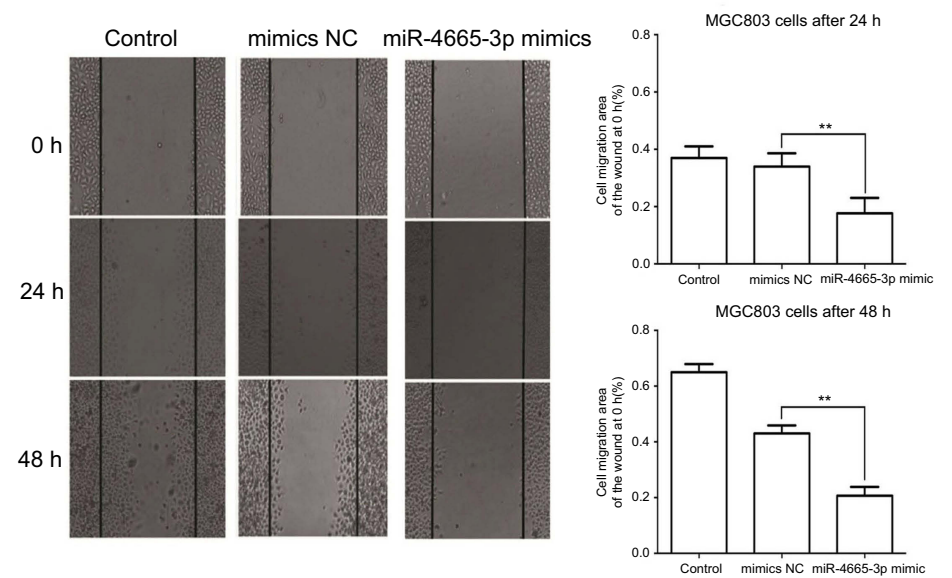

C

AGS

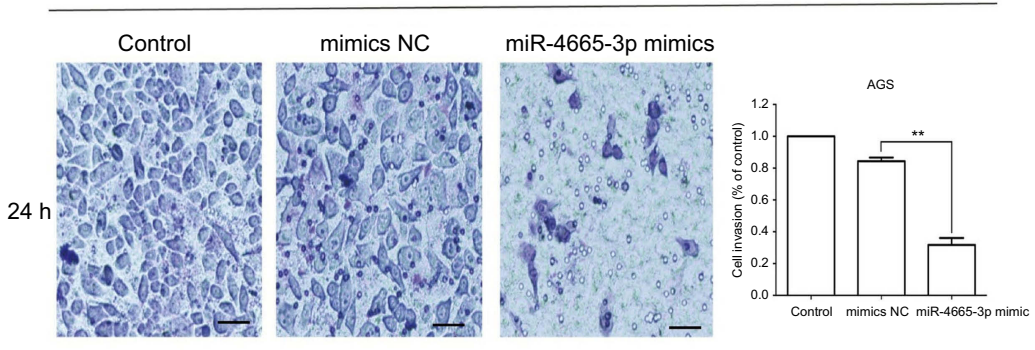

\section{D}

MGC803

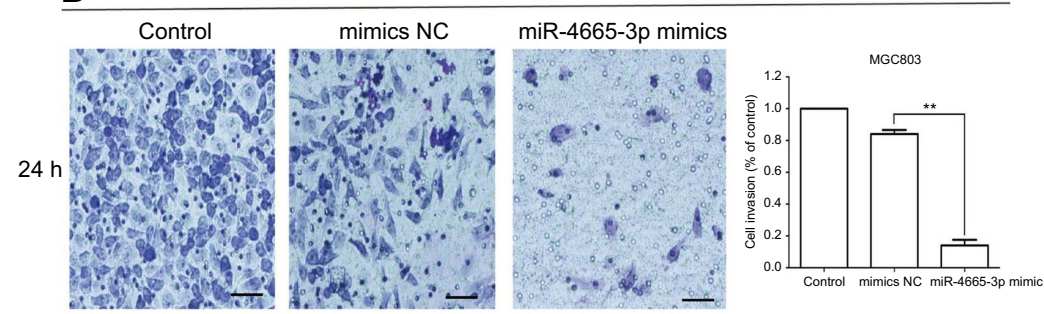

Figure 4 MiR-4665-3p inhibits migration and invasion of gastric cancer cells.

Notes: (A) AGS cells were scratched after transfected with mimics NC, miR-4665-3p mimics for $24 \mathrm{~h}$. (B) MGC803 cells were scratched after transfected with mimics NC, miR4665-3p mimics for $24 \mathrm{~h}$. The migration was observed under a microscope at a magnification of $100 \times$ for $0,24,48 \mathrm{~h}$. (C) Cell invasion was determined in AGS cells transfected with mimics NC and miR-4665-3p mimics by Transwell invasion assay. (D) Cell invasion was determined in MGC803 cells transfected with mimics NC and miR-4665-3p mimics by transwell invasion assay. The transfected cells were cultured in the top part of a transwell chamber for $24 \mathrm{~h}$. Then cells at the lower part of the chamber were observed under a microscope at a magnification of $200 \times$. Scale bar, $100 \mu \mathrm{m}$. Data represents the mean \pm standard deviation of three independent experiments. $* * P<0.0 \mathrm{I}$. 
A

AGS

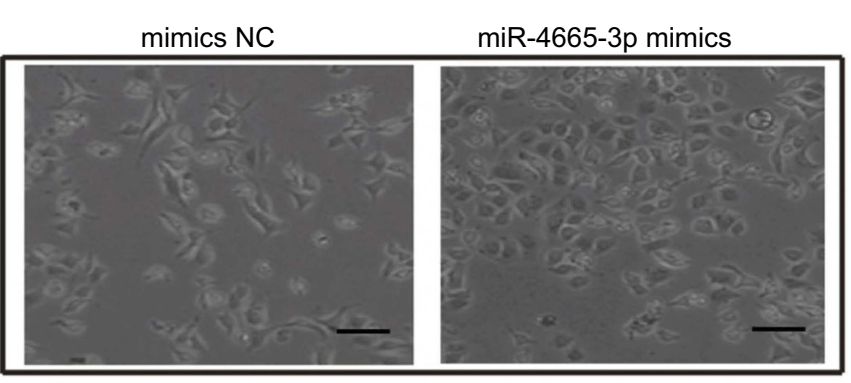

B

MGC803

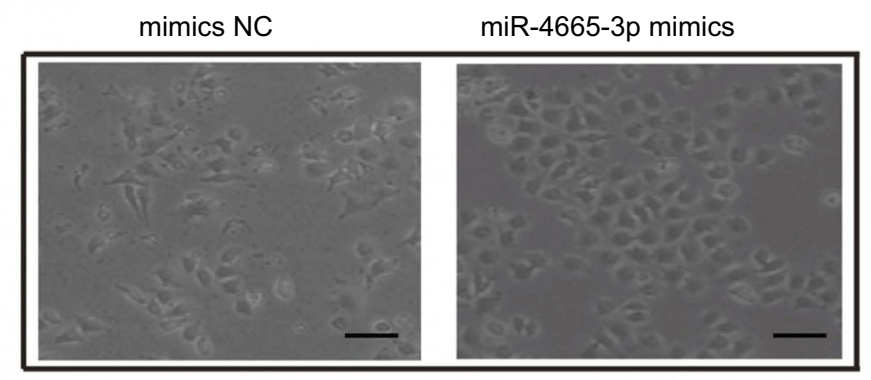

C

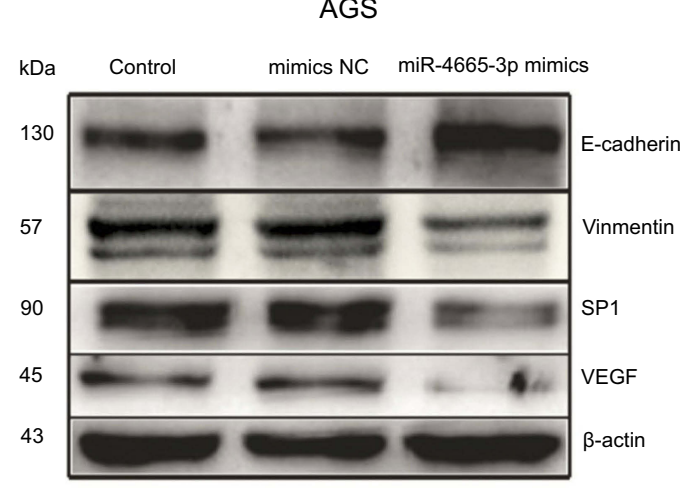

D

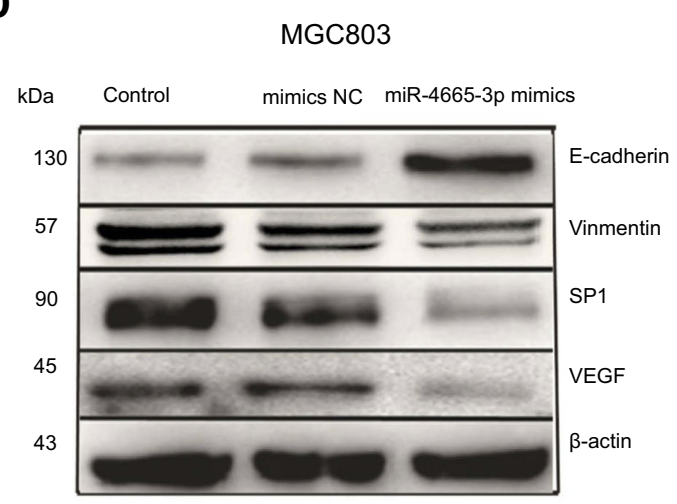

Figure 5 MiR-4665-3p over-expression reverses the EMT of GC cells.

Notes: (A) The morphology changes of AGS cells after treatment with miR-4665-3p mimics and mimics NC. Magnification, 200x. Scale bar, I00 $\mu$ m. (B) The morphology changes of MGC803 cells after treatment with miR-4665-3p mimics and mimics NC. Magnification, 200x. Scale bar, $100 \mu \mathrm{m}$. (C) The alteration of proteins associated with EMT in AGS cells after treatment with miR-4665-3p mimics. kDa, molecular weight. (D) The alteration of proteins associated with EMT in MGC803 cells after treatment with miR-4665-3p mimics. $\mathrm{kDa}$, molecular weight.

target genes is critical for revealing novel therapeutic targets of cancer treatment.

Here, we show for the first time that miR-4665-3p functions as a critical suppressor in the migration and invasion of GC cells. In this study, we firstly screened the miRNA expression of AGS cells which were treated with $\mathrm{As}_{4} \mathrm{~S}_{4}$ for $12 \mathrm{~h}$ through miRNA microarray. We then analyzed the alterations of the miRNAs between cells with or without $12 \mathrm{~h} \mathrm{As} \mathrm{S}_{4}$ incubation. Among the miRNAs whose fold-change exceeds two folds, the up-regulation of miR-4665-3p, miR-4739, miR-135b-5p, and the down- regulation of miR-6124 were further verified through qRT-PCR, which was consistent with the microarray analysis. We previously reported that $\mathrm{As}_{4} \mathrm{~S}_{4}$ exerts anti-cancer properties by inhibiting the proliferation, migration and invasion of GC cells. ${ }^{19}$ The miRNAs which are up-regulated by $\mathrm{As}_{4} \mathrm{~S}_{4}$ are likely to exert anti-cancer properties while the down-regulated one may be carcinogenic. MiRNAs with anticancer properties are often lowly expressed in cancer tissues. Meanwhile, the carcinogenic ones are often highly expressed. To better investigate the functions of these miRNAs and their roles in the 

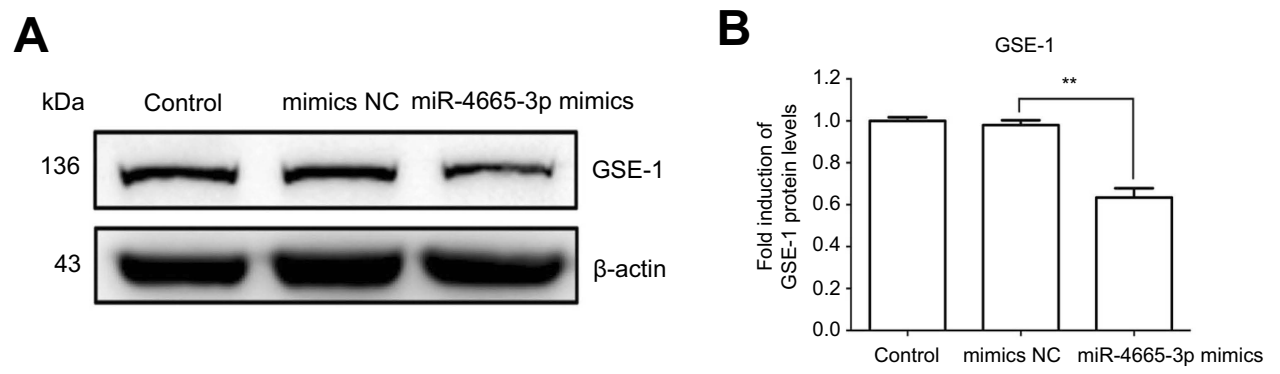

Figure 6 GSEI is a direct target of miR-4665-3p in gastric cancer cells.

Notes: (A) The protein level of GSEI was examined using Western blotting in AGS cells transfected with miR-4665-3p, NC mimics was used as a control. kDa, molecular weight. (B) The quantitative analysis of the relative density of GSE-I compared with $\beta$-actin is shown on the right panel as indicated. Data represents one of the three identical experiments with similar results. $* * P<0.01$

Table 3 The analysis diagram of the sequence complementary relationship between miR-4665-3p and GSEI

\begin{tabular}{|c|c|c|c|c|}
\hline \multicolumn{5}{|l|}{ GSEI } \\
\hline \multicolumn{5}{|c|}{ NM_0I46I5-3utr(mir-4665-3p) } \\
\hline \multicolumn{5}{|c|}{ CAGAACTGGACCACTTACGAAAGTGCCTTGCCTTGCCTGCAATGCACTGGCCTAGGGGCTACCTGAAGGGATATCCCAGGTGACGGTT } \\
\hline \multicolumn{5}{|c|}{ TCCCTTGCACTAGGCCGAACCTATAGTATAGAAATATTATCTATTTTATTACCTTGAATATTTAATATTTTTTCACTGGGAGGTTTGAAGCTTAC } \\
\hline \multicolumn{5}{|c|}{ AAAATGAGAATGTGCCATGCATGA } \\
\hline \multicolumn{5}{|c|}{ NM_0146I5-3utr(mir-4665-3p)-mut } \\
\hline \multirow{3}{*}{\multicolumn{5}{|c|}{$\begin{array}{l}\text { CAGAACTGGACCACTTACGAAAGTGCCTTGCCTTGCCTGCAATGCACTGGCCTAGGGGCTACCTGAAGGGATATCCCAGGTGACGGTT } \\
\text { TCCCTTGCACTATTAATCACCTATAGTATAGAAATATTATCTATTTTATTACCTTGAATATTTAATATTTTTCACTGGGAGGTTTGAAGCTTACA } \\
\text { AAATGAGAATGTGCCATGCATGA }\end{array}$}} \\
\hline & & & & \\
\hline & & & & \\
\hline & $\begin{array}{l}\text { Predicted consequential pairing of target region (top) and } \\
\text { miRNA (bottom) }\end{array}$ & $\begin{array}{l}\text { Site } \\
\text { type }\end{array}$ & $\begin{array}{l}\text { Context++ } \\
\text { score }\end{array}$ & $\begin{array}{l}\text { Context++ score } \\
\text { percentile }\end{array}$ \\
\hline $\begin{array}{l}\text { Position 18-24 of GSE1 } 13^{\prime} \text { UTR } 5 \\
\text { hsa-miR-4665-3p }\end{array}$ & $\begin{array}{l}\text {... GUUUJCCCUUGCACUAGGCCGAAC... } \\
|||||| \mid \\
\text { CСGCCCCCCGAUGCGCGGCGCCGGCUC }\end{array}$ & $\begin{array}{c}7 \mathrm{mer}- \\
\mathrm{A} 1\end{array}$ & -0.30 & 93 \\
\hline
\end{tabular}

progression of $\mathrm{GC}$, we next detected the expression of these miRNAs between 30 pairs of GC tissues and the adjacent normal tissues. We found that the expression of miR-4665-3p was obviously lower in GC tissues than paired adjacent normal tissues. Noteworthily, the expression of miR-4665-3p is further reduced in late-stage GC tissues compared with the early-stage ones (Figure 3B), indicating that miR-4665-3p involves in the regulation of cell migration and invasion. By performing wound healing assay and Transwell invasion assay with GC cells transfected with miR-4665-3p mimics, we found that miR4665-3p acts as a suppressor upon the migration and invasion of $\mathrm{GC}$ cells.

EMT refers to the biological process by which epithelial cells lose their polarity and transform into a mesenchymal phenotype through certain procedures. ${ }^{24,25}$ This highly complex process is regulated by the extracellular matrix, autocrine, or paracrine cell factors as well as miRNAs. ${ }^{8,17}$ The epithelial cells gradually lose their connection with the basement membrane during the process of EMT, and meanwhile, they also acquire the migratory properties. $^{21}$ The loss of the cell-cell adhesion and disruption of cell-cell junctions promotes the cell to migrate and invade easily and thus give rise to the metastasis of cancer. Previous studies indicated that the miR-15/107 group of miRNA genes regulate the EMT and migration invasion of tumors. ${ }^{26}$ Accumulating evidence proves that miRNAs are also involved in the regulation of EMT in GC, including miR-125a, miR-618 and miR-1254. ${ }^{16-18}$ Furthermore, EMT is typically characterized by decreased expression of cell adhesion proteins, such as E-cadherin, while it is increased in mesenchymal-associated molecules, such as VEGF, Vimentin and SP1. E-cadherin is a key mediator of cell-cell adhesions in epithelial tissues, and loss of E-cadherin can promote invasive and metastatic characteristics in many epithelial tumors. Vimentin is a cytoskeletal protein, the abnormal expression of vimentin was also observed in a variety of epithelial tumors and had a close relationship with differentiation, invasion and metastasis of cancer cell. ${ }^{27}$ As one of the most effective factors which can induce angiogenesis, there is solid evidence suggesting that the expression of VEGF is closely related 
to tumor metastasis and prognosis. ${ }^{28,29}$ Additionally, the upstream regulator SP1 could bind to the promoter site of VEGF and further manipulate tumor angiogenesis and metastasis. Our data indicated that mesenchymal characteristics were reduced after the upregulation of miR-4665$3 p$ in AGS cells and MGC803 cells. Through the morphological observation, we found that GC cells transfected with miR-4665-3p underwent morphological changes from an extended morphology to more organized cellcell contacts. As shown in Figure $5 \mathrm{~A}$ and $\mathrm{B}$, the cell morphology showed an epithelialization trend with the cell morphology changes from spindle-shaped to cobblestone-shaped. We then further verified that overexpressed miR-4665-3p could suppress EMT by dramatically increasing levels of E-cadherin, while downregulating the expression of VEGF, Vimentin and SP1. These findings showed consistency with our migration and invasion results.

The anti-tumor property of miRNAs is known to inhibit cancer development by upregulating tumor suppressor genes or silencing oncogenes. To clarify the mechanisms underlying the effects of miR-4665-3p on migration, and invasion. Bioinformatics analysis was used to predict putative targets of miR-4665-3p in GC cells. Among the candidate target genes, we concentrated on GSE1. Human Gse1 coiled-coil protein (GSE1), also known as KIAA0182, is a proline-rich protein. ${ }^{30}$ Recent studies have demonstrated that GSE1 possesses an oncogenic function in human breast cancer cells and it is negatively regulated by miR-489-5p and promotes both proliferation and metastasis in breast cancer cells. ${ }^{31}$ Further, GSE1 promoted cell proliferation, colony formation, migration, invasion, tumor growth, and metastasis both in vitro and in vivo of GC. Moreover, gastric cancer patients with high tumor expression of GSE1 exhibited worse clinicopathological parameters and survival rates. ${ }^{22}$ And here we observed that the expression of GSE1 could be decreased by miR-4665-3p. On the basis of these data, we speculated that $\mathrm{miR}-4665-3 p$ may interfere with the progression of tumor metastasis of GC through the inhibition of the GSE1.

In conclusion, our results showed that miR-4665-3p was lowly expressed in GC tissues. Overexpression of miR-4665-3p induced by $\mathrm{As}_{4} \mathrm{~S}_{4}$ abrogated GC cells migration, invasion by binding and down-regulating the tumor oncogene GSE1. The newly identified inhibitory effect of miR-4665-3p upon GC cells provides new insight into the pathogenesis of $\mathrm{GC}$ and presents a potential novel biomarker. Therefore, our research provides deeper descript of the molecular mechanism of GC progression.

\section{Acknowledgments}

This work was supported by the National Science Foundation of China (81874353 and 81274142), and the Science and Technology Commission of Shanghai Municipality (17401931300 and 11ZR1423400).

\section{Disclosure}

The authors report no conflicts of interest in this work.

\section{References}

1. Siegel RL, Miller KD, Jemal A. Cancer statistics, 2019. CA Cancer J Clin. 2019;69(1):7-34. doi:10.3322/caac.21551

2. Ferlay J, Shin H-R, Bray F, Forman D, Mathers C, Parkin DM. Estimates of worldwide burden of cancer in 2008: GLOBOCAN 2008. Int J Cancer. 2010;127(12):2893-2917. doi:10.1002/ijc.25516

3. Wagner AD, Grothe W, Haerting J, Kleber G, Grothey A, Fleig WE. Chemotherapy in advanced gastric cancer: a systematic review and meta-analysis based on aggregate data. J Clin Oncol. 2006;24 (18):2903-2909. doi:10.1200/JCO.2005.05.0245

4. Houghton J, Wang TC. Helicobacter pylori and gastric cancer: a new paradigm for inflammation-associated epithelial cancers. Gastroenterology. 2005;128(6):1567-1578.

5. Zambon C-F, Basso D, Navaglia F, et al. Increased risk of noncardia gastric cancer associated with proinflammatory cytokine gene polymorphisms. Gastroenterology. 2004;126(1):382-384.

6. Yamaguchi N, Kakizoe T. Synergistic interaction between Helicobacter pylori gastritis and diet in gastric cancer. Lancet Oncol. 2001;2(2):88-94. doi:10.1016/S1470-2045(00)00225-4

7. Eun JW, Kim HS, Shen Q, et al. MicroRNA-495-3p functions as a tumor suppressor by regulating multiple epigenetic modifiers in gastric carcinogenesis. J Pathol. 2018;244(1):107-119. doi:10.1002/ path. 4994

8. Lei C, Du F, Sun L, et al. miR-143 and miR-145 inhibit gastric cancer cell migration and metastasis by suppressing MYO6. Cell Death Dis. 2017;8(10):e3101. doi:10.1038/cddis.2017.518

9. Ambros V. The functions of animal microRNAs. Nature. 2004;431 (7006):350-355. doi:10.1038/nature02871

10. Bartel DP. MicroRNAs: genomics, biogenesis, mechanism, and function. Cell. 2004;116(2):281-297.

11. Fire A, Xu S, Montgomery MK, Kostas SA, Driver SE, Mello CC. Potent and specific genetic interference by double-stranded RNA in Caenorhabditis elegans. Nature. 1998;391(6669):806-811. doi:10. $1038 / 35888$

12. Yu Z, Jian Z, Shen SH, Purisima E, Wang E. Global analysis of microRNA target gene expression reveals that miRNA targets are lower expressed in mature mouse and Drosophila tissues than in the embryos. Nucleic Acids Res. 2007;35(1):152-164. doi:10.1093/nar/ gk11032

13. Lewis BP, Burge CB, Bartel DP. Conserved seed pairing, often flanked by adenosines, indicates that thousands of human genes are microRNA targets. Cell. 2005;120(1):15-20. doi:10.1016/j.cell.2004. 12.035

14. Calin GA, Croce CM. MicroRNA signatures in human cancers. Nat Rev Cancer. 2006;6(11):857-866. doi:10.1038/nrc1997

15. Garofalo M, Croce CM. microRNAs: master regulators as potential therapeutics in cancer. Annu Rev Pharmacol Toxicol. 2011;51:25-43. doi:10.1146/annurev-pharmtox-010510-100517 
16. Yang L, Zhang S, Guo K, et al. miR-125a restrains cell migration and invasion by targeting STAT3 in gastric cancer cells. Onco Targets Ther. 2019;12:205-215. doi:10.2147/OTT.S168454

17. Shi J, Gong L, Chen L, et al. MiR-618 suppresses metastasis in gastric cancer by downregulating the expression of TGF-beta2. Anat Rec (Hoboken). 2019. doi:10.1002/ar.v302.6

18. Jiang M, Shi L, Yang C, et al. miR-1254 inhibits cell proliferation, migration, and invasion by down-regulating Smurf1 in gastric cancer Cell Death Dis. 2019;10(1):32. doi:10.1038/s41419-019-1300-3

19. Zhang L, Kim S, Ding W, et al. Arsenic sulfide inhibits cell migration and invasion of gastric cancer in vitro and in vivo. Drug Des Devel Ther. 2015;9:5579-5590. doi:10.2147/DDDT.S89805

20. Zhang L, Tian W, Kim S, Ding W, Tong Y, Chen S. Arsenic sulfide, the main component of realgar, a traditional Chinese medicine, induces apoptosis of gastric cancer cells in vitro and in vivo. Drug Des Devel Ther. 2015;9:79-92. doi:10.2147/DDDT.S74379

21. Polyak K, Weinberg RA. Transitions between epithelial and mesenchymal states: acquisition of malignant and stem cell traits. Nat Rev Cancer. 2009;9(4):265-273. doi:10.1038/nrc2620

22. Ding K, Tan S, Huang X, et al. GSE1 predicts poor survival outcome in gastric cancer patients by SLC7A5 enhancement of tumor growth and metastasis. J Biol Chem. 2018;293(11):3949-3964. doi:10.1074/ jbc.RA117.001103

23. Farazi TA, Spitzer JI, Morozov P, Tuschl T. miRNAs in human cancer. J Pathol. 2011;223(2):102-115. doi:10.1002/path.2806

24. Brabletz T, Kalluri R, Nieto MA, Weinberg RA. EMT in cancer. Nat Rev Cancer. 2018;18(2):128-134. doi:10.1038/nrc.2017.118
25. Kim NH, Kim HS, Li X-Y, et al. A p53/miRNA-34 axis regulates Snaill-dependent cancer cell epithelial-mesenchymal transition. $J$ Cell Biol. 2011;195(3):417-433. doi:10.1083/jcb.201103097

26. Martello G, Rosato A, Ferrari F, et al. A MicroRNA targeting dicer for metastasis control. Cell. 2010;141(7):1195-1207. doi:10.1016/j. cell.2010.05.017

27. Zhou J, Tao D, Xu Q, Gao Z, Tang D. Expression of E-cadherin and vimentin in oral squamous cell carcinoma. Int J Clin Exp Pathol. 2015;8(3):3150-3154.

28. Yu XM, Lo CY, Chan WF, Lam KY, Leung P, Luk JM. Increased expression of vascular endothelial growth factor $\mathrm{C}$ in papillary thyroid carcinoma correlates with cervical lymph node metastases. Clin Cancer Res. 2005;11(22):8063-8069. doi:10.1158/1078-0432.CCR05-0646

29. Lee SH, Lee SJ, Jin SM, et al. Relationships between lymph node metastasis and expression of CD31, D2-40, and vascular endothelial growth factors A and C in papillary thyroid cancer. Clin Exp Otorhinolaryngol. 2012;5(3):150-155. doi:10.3342/ceo.2012.5. 3.150

30. Hakimi MA, Dong Y, Lane WS, Speicher DW, Shiekhattar R. A candidate $\mathrm{X}$-linked mental retardation gene is a component of a new family of histone deacetylase-containing complexes. J Biol Chem. 2003;278(9):7234-7239. doi:10.1074/jbc.M208992200

31. Chai P, Tian J, Zhao D, et al. GSE1 negative regulation by miR-489$5 \mathrm{p}$ promotes breast cancer cell proliferation and invasion. Biochem Biophys Res Commun. 2016;471(1):123-128. doi:10.1016/j.bbrc. 2016.01.168 


\section{Supplementary material}

\section{MGC803}
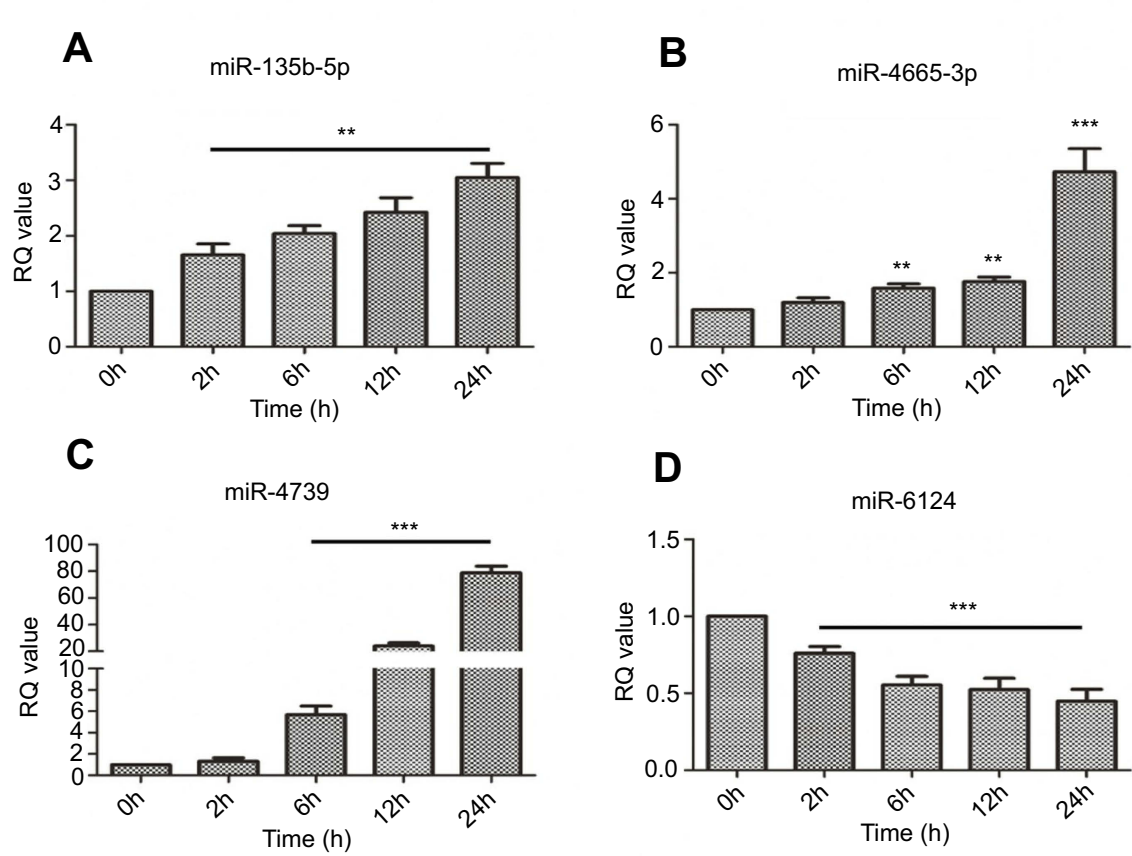

\section{HCT116}
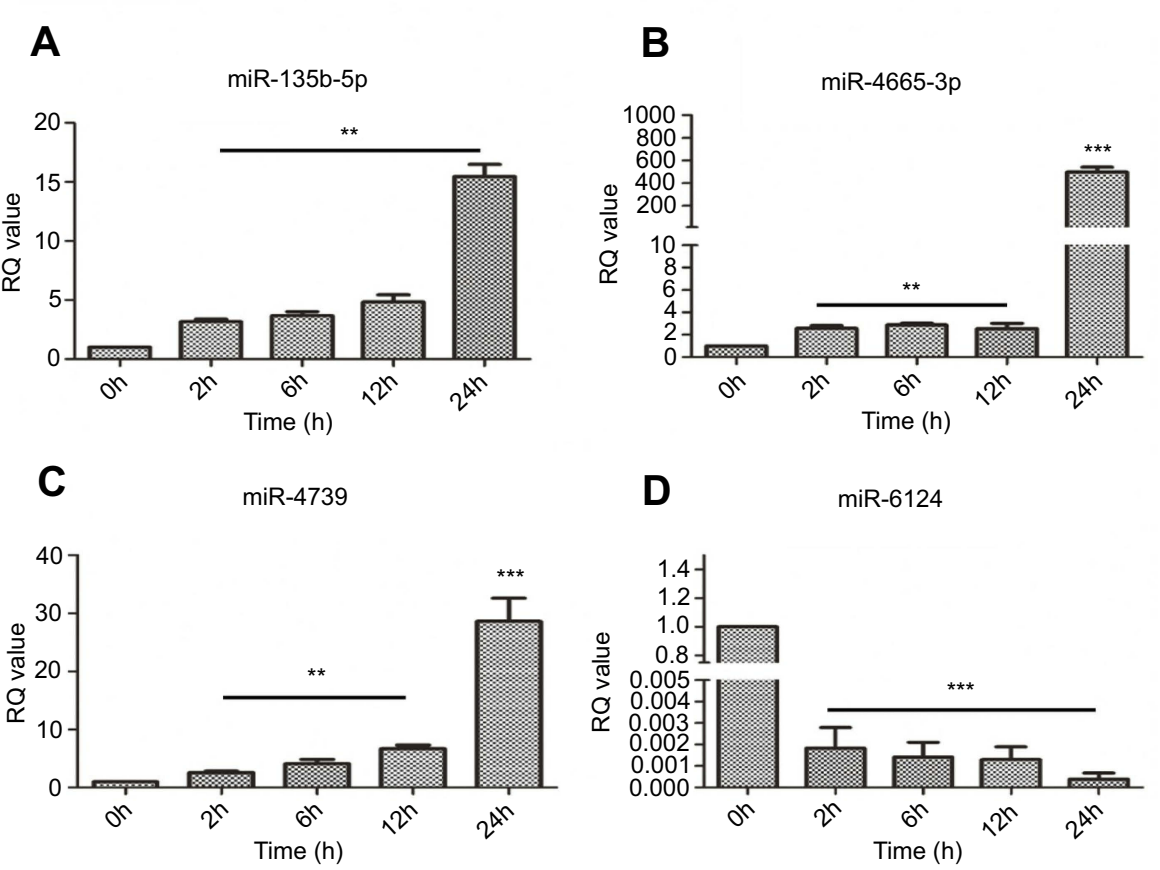

Figure SI The alteration of miRNAs after treated with $\mathrm{As}_{4} \mathrm{~S}_{4}$ in MGC803 and HCTII6.

Notes: (A) miR-135b-5p, (B) miR-4665-3p, (C) miR-4739, (D) miR-6I24. MiR-I35b-5p, miR-4665-3p and miR-4739 are up-regulated while miR-6I24 is down-regulated after incubation with $\mathrm{As}_{4} \mathrm{~S}_{4}$ in both $\mathrm{MGC} 803$ cells and HCTII6 cells. $* * P<0.01$, $* * * P<0.00 \mathrm{I}$. 


\section{Publish your work in this journal}

Drug Design, Development and Therapy is an international, peerreviewed open-access journal that spans the spectrum of drug design and development through to clinical applications. Clinical outcomes, patient safety, and programs for the development and effective, safe, and sustained use of medicines are a feature of the journal, which has also

been accepted for indexing on PubMed Central. The manuscript management system is completely online and includes a very quick and fair peer-review system, which is all easy to use. Visit http://www. dovepress.com/testimonials.php to read real quotes from published authors.

Submit your manuscript here: https://www.dovepress.com/drug-design-development-and-therapy-journal 